EUROPEAN ORGANIZATION FOR NUCLEAR RESEARCH

CERN-EP/88-41

March 24th, 1988

\title{
LEAD BEAM EXPERIMENTS AT THE CERN SPS
}

\author{
A. SANDOVAL \\ GSI, D - 6900 Darmstadt, West Germany \\ and \\ CERN, Geneva, Switzerland \\ Presented at the Quark Matter 87 Conference \\ Nordkirchen, West Germany \\ 23-29 August 1987
}

\section{Abstract:}

The acceleration in 1986 of ${ }^{16} \mathrm{O}$ beams to $200 \mathrm{GeV} /$ nucleon at the CERN SPS created a new frontier of particle and nuclear physics, namely the study of high energy density systems with hundreds of quarks and gluons created in the central collisions of nuclei with heavy targets. In order to produce the largest piece of "quark matter" beams as heavy as $208 \mathrm{~Pb}$ are needed. The Lead - Injector Project that would provide them is presented. Possible experimental approaches to extract the physics from collisions with thousands of produced particles are discussed. 


\section{INTRODUCTION}

The Standard Model predicts that at high enough energy density nuclear matter undergoes a phase transition into a deconfined plasma of quarks and gluons in which chiral symmetry is restored [1]. The study of such a transition is not only academic, since the Universe probably underwent such a transition in the opposite direction when in the very early stages after the big-bang the energy density fell below a few $\mathrm{GeV} / \mathrm{fm}^{3}$. At that moment the deconfined quark-gluon plasma started to hadronize. The chemical potential of the different quark flavors at the transition stage and the hadronization mechanism probably played a significant role in determining the ratio of matter to antimatter as well as the fraction of baryons and mesons in the resulting hadronic state. Since from the quark-antiquark combinatorics at the moment of confinement, one expects far more mesons than baryons to be produced and since a fraction of the meson energy eventually ends up in the form of neutrinos, the actual path that was followed during hadronization is relevant to the missing mass problem and to questions of whether the Universe is open or closed.

The flavor composition of a plasma in equilibrium and the energy sharing between quarks, gluons, photons and leptons are questions that for the moment have no answer. The relevant theoretical tool, Lattice QCD is not yet advanced enough to treat such a complicated system with sufficient predictive power. By comparison with electromagnetic plasmas where one has only photons and negative and positive charges, the variety and complexity of the phenomena that one would expect in quark matter will be much greater since in the QCD case one has colored, fractionally charged quarks and antiquarks in up to 6 flavors, eight different types of self interacting gluons, photons, leptons and intermediate vector bosons.

The availability of ultrarelativistic nuclear beams since the end of 1986 at CERN and Brookhaven has opened up a new field of physics to systematic experimental study. The large number of results and their quality reported at this conference hardly one year after the first beams were available shows with what intensity this new field is being pursued. The study of transverse encrgy production [2], [3], [4] has shown that in central collision of $200 \mathrm{GeV} /$ nucleon 160 with heavy targets one reaches in a system with a large number of quarks and gluons, although only for a brief instant of time, energy densities of the order of $3 \mathrm{GeV} / \mathrm{fm}^{3}$, that is, more than twenty times the energy density of ground state nuclear matter. The rapidity distributions of the produced particles in this type of events, [5], [6], [7] their transverse momentum distributions [5], [7], [8], [9] and the shape parameters of the pion emitting source measured by two pion interferometry [10] hint that a large fraction of the incoming energy is stopped in the collision and that at mid-rapidity there is a system that tends to thermal equilibrium emitting hundreds of pions. The observed effect of the melting of the $\mathrm{J} / \psi$ [9] might be due to the formation of a quark-gluon plasma. One might therefore see here the birth of experimental QCD Thermodynamics.

At the end of 1987 data was taken with a heavier projectile, ${ }^{32} \mathrm{~S}$, but it is clear that in order to make a 'macroscopic' piece of quark matter projectiles with the heaviest mass available $\left({ }^{208} \mathrm{pb}\right)$ are needed. The present injector Linac has reached with ${ }^{32} \mathrm{~S}$ its limit regarding the mass of the ion species it can accelerate. Therefore in order to provide still heavier nuclei a new accelerating structure is needed. Reinhard Stock has initiated the drive for such a development [11] which has been supported by the ion community crystalyzing into what is called the Lead-Injector Project [12]. The construction of such a facility will give CERN the highest energy ion beams over the whole periodic table. It will be a challenge for accelerator designers to build an injector optimized to the acceptance, momentum swing, bunching, etc. of the PS Booster, PS and SPS. For the experimental physicists it will be an even greater challenge to design instruments that can extract the physics of interactions in which several thousand particles are produced.

In this paper the present status of the accelerator scheme will be presented and some of the detectors one might use in the lead era will be discussed. 


\section{THE LEAD INJECTOR}

The major constraint for accelerating heavier ions than ${ }^{32} \mathrm{~S}$ in the CERN accelerator complex is the old Linac 1 which can only accelerate partially stripped ions with a charge to mass ratio Q/A > 0.35 . Since at least $30 \mu \mathrm{A}$ are needed from the injector to have enough intensity to monitor the beam in the synchrotrons, the ion source technology is already pushed to the limit in order to provide enough current of $S(12+)$. Higher masses with $Q / A>0.35$ cannot be attained with sufficient yield.

The acceleration of heavy projectiles thus calls for replacement of the Linac 1 by a structure accelerating ions with $0.15<\mathrm{Q} / \mathrm{A}<0.2$ which corresponds to $\mathrm{Pb}(30+)$ to $\mathrm{Pb}(40+)$. This requires new developments in ECR ion source technology, a new RFQ (radio frequency quadrupole) and a new Linac structure that constitutes the major cost factor [12], [13]. A sketch of such an injector structure is shown in Fig. 1. By using a high charge state source and a correspondingly short linac the injector will fit into the available space without new building construction. The PS - Booster and PS synchrotrons will have to accelerate incompletely stripped ions. This requires an average vacuum level of $10^{-9}$ Torr which is available at the PS but calls for an upgrade program at the Booster. At the SPS no additional effort will be required other than the implementation of a rebunching acceleration mode. The top SPS extraction energy for $208 \mathrm{pb}$ will be $180 \mathrm{GeV} /$ nucleon. The new facility will provide external beams of all lighter nuclei as well, with a maximum energy of $225 \mathrm{GeV} /$ nucleon up to ${ }^{40} \mathrm{Ca}$, and external beam intensities of about $5 \times 10^{8}$ ions $/ \mathrm{s}(\mathrm{Ca})$ to $10^{7} \mathrm{ions} / \mathrm{s}(\mathrm{Pb})$. The total cost is about $30 \mathrm{MSF}$.

If CERN constructs the Large Hadron Collider (LHC) in the 1990's as a next project after LEP, one would also do colliding nuclei at center of mass energies of 3 to $4 \mathrm{TeV} /$ nucleon. In order to reach luminosities of $2 \times 10^{26}$ one would need a factor of 10 higher intensities injected into the Booster. A possible solution for such an injector is also shown in the lower part of Fig. 1, which would mean an additional cost of $20 \mathrm{MSF}$. An optimized solution that would satisfy both requirements is being studied at present.

\section{WHAT WE LEARNED ABOUT THE INSTRUMENTATION IN THE FIRST EXPERIMENTS}

Up to the end of 1986 before the first round of experiments was completed at CERN and Brookhaven, many people had serious doubts that one could measure single particle differential quantities in events where several hundred particles are produced. One thought that only calorimeters would be able to cope with the particle density by measuring the transverse energy flow into certain regions of laboratory pseudorapidity. As the first experiments have shown this turned out not to be the case. Differential quantities have been measured for many probes, among them:

1. The measurement of high mass muon pairs which show a clear $\psi$ peak enabling one to measure their $\mathrm{p}_{\mathrm{T}}$ distribution at mid-rapidity for different types of collisions tagged by a midrapidity calorimeter [9].

2. The measurement of $\Lambda, \Lambda$ and $\mathrm{K}^{0}$ production over a large range of $\mathrm{y}$ and $\mathrm{pT}$ from their charged particle decay in the fiducial volume of a Streamer Chamber [14].

3. The determination of $\pi^{+}, \pi^{-}, \mathrm{K}^{+}, \mathrm{K}^{-}$and $\mathrm{p}$ momentum distributions over selected ranges of laboratory angles [7], [15].

4. The measurement of $\gamma \mathrm{p}_{\mathrm{T}}$ distributions both with a fine grained electromagnetic calorimeter [8] and by the conversion method in a multiparticle spectrometer [16].

5. The measurement of mid-rapidity $\pi^{0} \mathrm{p}_{\mathrm{T}}$ distributions reconstructed from $\gamma-\gamma$ invariant mass distributions [8]. 


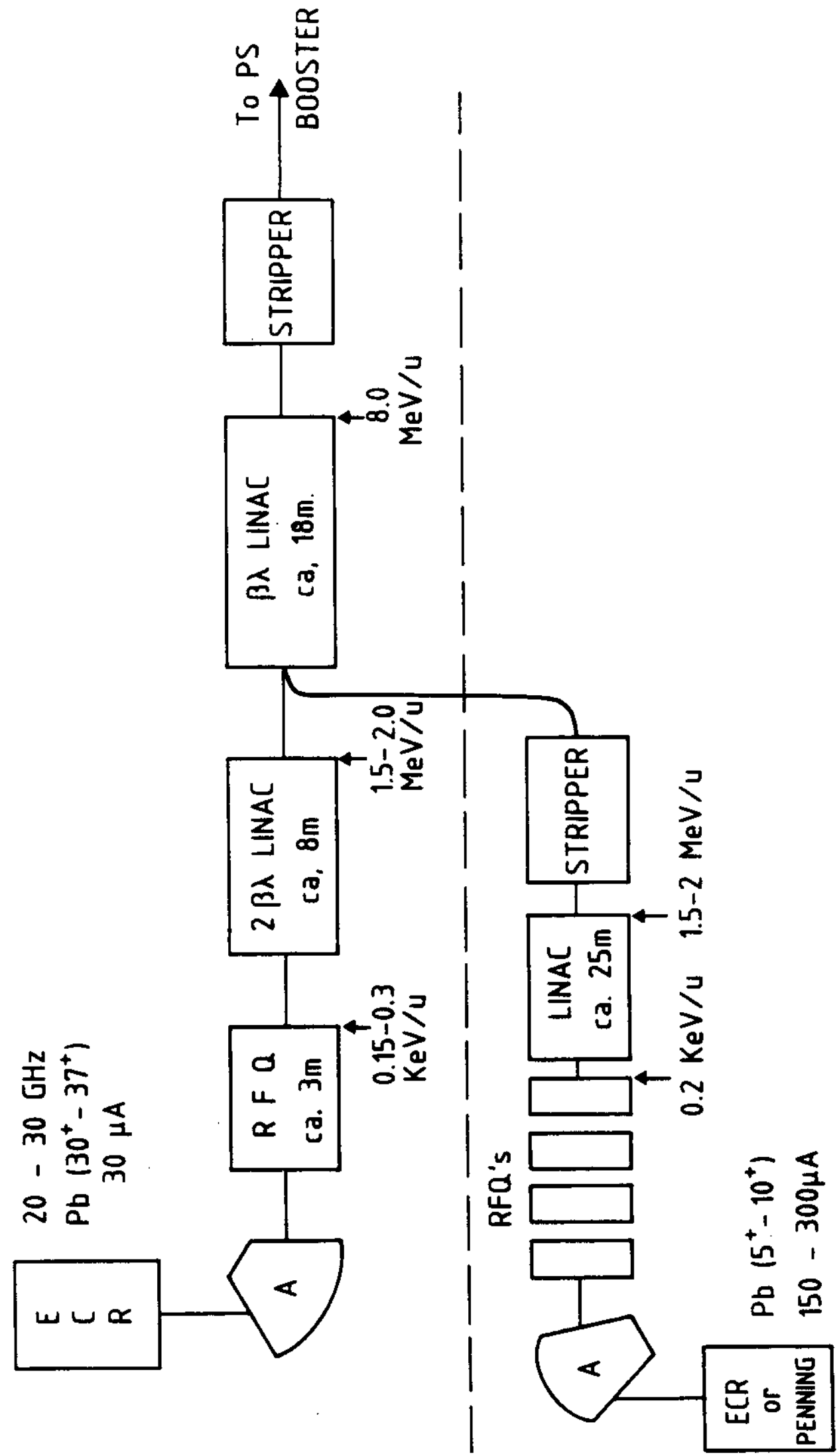

Figure 1. Sketch of the injector structure to provide ions of any mass up to $\mathrm{Pb}$ for the CERN accelerator complex. In the upper haif a high charge state, low current solution for fixed target experiments. In the lower half a low charge state high current solution for colliding bearns in the LHC. 
6. The measurement of the rigidity of all the charged particles emitted in an interaction, leading to $\mathrm{y}$ and $\mathrm{p}_{\mathrm{T}}$ distributions for $\pi^{-}$and to the determination of the shape parameters of the pion emitting source through two-pion interferometry [5], [10].

7. The measurement of target rapidity nuclear fragments [17].

8. The measurement of the differential multiplicity distributions as a function of pseudorapidity [6], [18], [19], [20].

9. The measurement of differential $\mathrm{E}_{\mathrm{T}}$ distributions as a function of pseudorapidity [2], [3], [4].

As can be seen most of the efforts made to measure single particle differential quantities succeeded inspite of the high multiplicity of produced particles. Can the same type of measurements be made with the present instrumentation for central collisions of heavier beams up to $\mathrm{Pb}$, where one expects to produce about 3000 charged particles and as many gammas? The answer for most of the cases is no. The present detectors will be swamped to a point where the individual particles can no longer be reconstructed. The clear exception is calorimetry where the higher particle density only improves the measurement of the energy flux by averaging out the inherent fluctuations.

Is it necessary to design and build a second generation experiment for the Lead - era where measurements of single particle observables are possible, or is it sufficient to do calorimetry in a way not much different to what the present experiments do? It will be argued that in order to exploit the physics of ultrarelativistic ion collisions it is absolutely essential to build a second generation experiment to do single particle differential measurements and what is even more important one needs to determine the full four dimensional momentum vector of the detected particles.

The physics aim of this field is at present to study and understand the behavior of a system of hundreds to thousands of quarks and gluons as they go from the ground state hadron phase of the initial nuclei, through a high energy density phase where hundreds of new partons are created, into an expanding system where hadronization takes place, followed by the decay of heavy clusters into the experimentally observable final state. It is clear that in order to understand such a complicated process one has to study not only exotic probes, but also the majority of the produced particles. One would like to know how the energy is partitioned, what is the flavor composition as a function of $y$ and PT, what is the yield of low mass lepton pairs, of $\phi^{\prime} \mathrm{s}$, of $\psi^{\prime} \mathrm{s}$, of $\mathrm{N}$, etc., how is the charge and strangeness balanced in $\mathrm{y}$ and $\mathrm{p}_{\mathrm{T}}$, what are the shapes of the pion, kaon and proton emitting sources and all these as a function of an observable related to the maximum energy density reached in the interaction like the produced transverse energy. To do this one needs to identify the particles and measure their momenta over a given part of phase space. Since one needs to do a systematic study of all these observables for different projectile - target combinations at various bombarding energies in order to search for trends that might signal a phase transition into a different state of quark matter and since these changes might be relatively small, one needs high precision measurements. Unfortunately calorimeters and similar type of detectors that measure observables as function of laboratory pseudorapidity $\eta$ can only be interpreted through folding of the model predictions with a Monte Carlo procedure. This reduces their assaying power.

The requirements for a second generation experiment therefore are extremely high and at present it is not clear that they can be met. A quick survey of some of the available options is given here by dividing the experiments into three broad categories:

- Di-muon measurements.

- Small acceptance spectrometers.

- Large solid angle detectors. 


\section{DI - MUON MEASUREMENTS}

An essential requirement for the detection of muon pairs is to have a large absorber very close to the target in order to stop immediately after production all $\pi$ 's and $\mathrm{K}^{\prime} \mathrm{s}$ before they have a chance to decay creating a larger muon background. This makes dimuon measurements incompatible with any other precision measurement and therefore they are treated as a separate class of experiments. The present series of measurements of NA34 and NA38 have shown that already with ${ }^{16} \mathrm{O}$ beams it is practically impossible to resolve the low mass resonances: $\eta, \rho, \omega, \phi$ 's due to the high combinatorial background. In contrast the $\mathrm{J} / \psi$ region is very clean as is seen in Fig. 2 . It would seem that with still heavier ions one could do very interesting measurements in this area. The effect of the increased combinatorial background and the problem of how to do an efficient trigger when each event produces several muons in the detector acceptance have to be investigated.

\section{SMALL ACCEPTANCE SPECTROMETERS}

Another strategy to measure the full kinematics of individual particles is to use small acceptance spectrometers. With them one could simultaneously measure and identify 10 or maybe up to 20 particles produced along a small solid angle. To mind come magnetic spectrometers with good momentum resolution that could measure charged particles at a very high rate. The data of the NA34 External Spectrometer and of Brookhaven experiment E802 show that such a solution can be applied to ion experiments. One could envision a setup of several spectrometers optimized to cover different parts of phase space. Such a system would then be complemented by calorimetry and multiplicity detectors to characterize the event.

The techniques involved are well understood and recent developments in fast trigger processors make the triggering on rare particles like $\mathrm{K}^{ \pm}$and $\mathrm{p}$ in such a multiparticle environment feasible. A configuration designed to study central $\mathrm{Pb}$ on $\mathrm{Pb}$ collisions should be investigated. Special attention has to be given to the background produced by the many simultaneously produced particles, some of which will shower in the magnets of the spectrometer.

\section{LARGE ACCEPTANCE DETECTORS}

Here a large acceptance detector is taken to be one that can simultaneously measure of the order of 100 particles in a selected part of phasespace. One can think of two different categories, one that measures charged particles with large magnetic fields, the other that measures gammas with large, fine grained electromagnetic calorimeters and reconstructs $\pi^{0} \mathrm{~s}$. A setup consisting of both types of detectors is discussed here.

One would start with a large vertex magnet that disperses the charged particles according to their charge and momentum over a much larger angular range than what they originally had, reducing the particle density for the tracking detectors, see Fig. 3. The tracking chambers would be placed downstream of the magnet at such a distance that the charged particle multiplicity is about 100 per $\mathrm{m}^{2}$ at the detector front face. In such a configuration the charged particle trajectories are almost parallel straight lines, making their three dimensional reconstruction much simpler than if one does the tracking in the magnetic field. Such a case is shown in Fig. 4, which shows a high multiplicity $\nu-F e$ interaction detected by the WA44 Avalanche Chamber. A 1 Tm magnet is used between target and detector. Clearly the tracking in such a situation is manageable.

From the position of the interaction vertex, the knowledge of the magnetic field and the track's 3 -dimensional position one obtains the momentum. In order to get enough resolution to do two- 


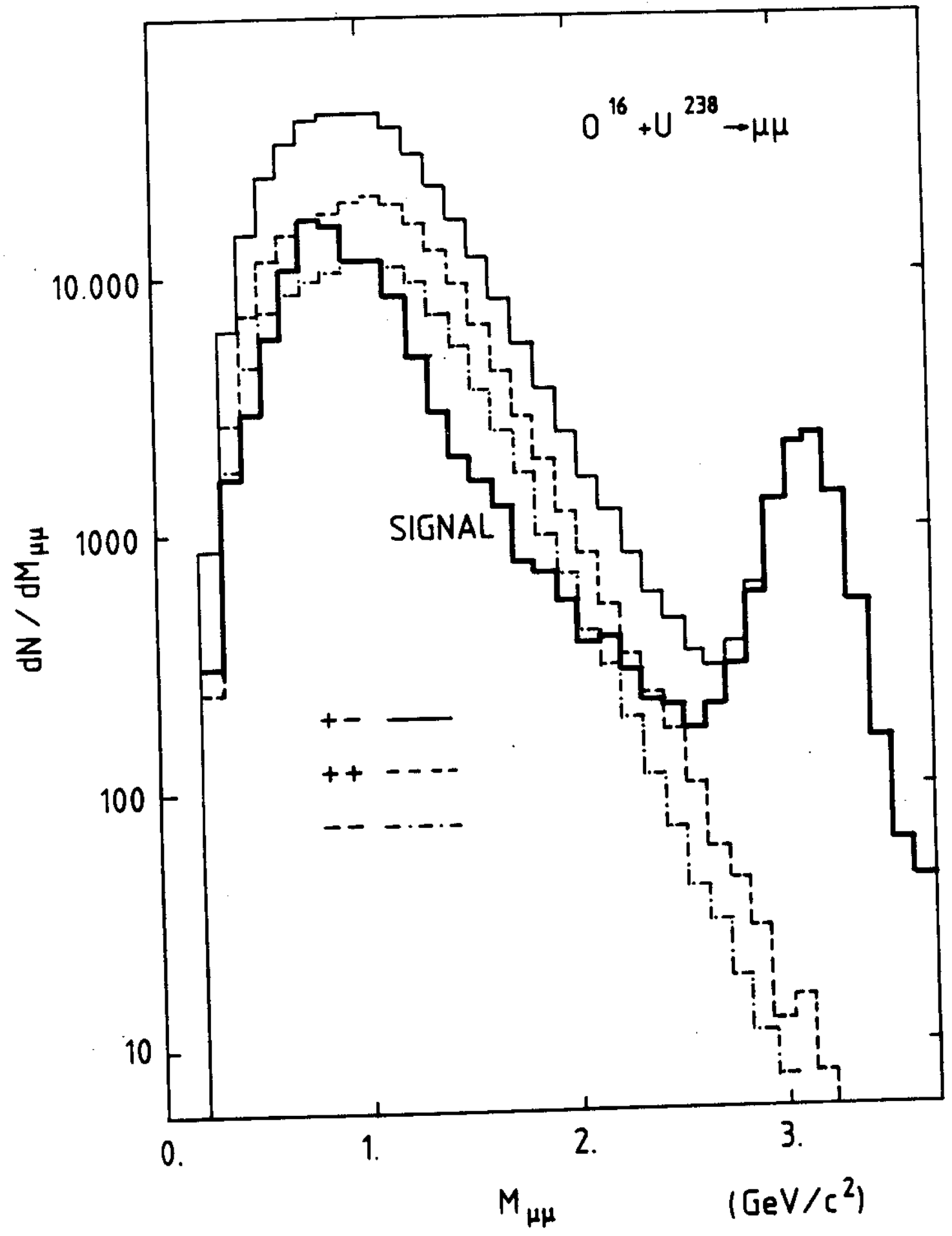

Fig. 2. Invariant mass distributions for muon-pairs measured by NA38 in $160+U$ collisions at $200 \mathrm{GeV} /$ nucleon. The region of the $\mathrm{J} / \psi$ is seen to be clean from combinatorial background. 

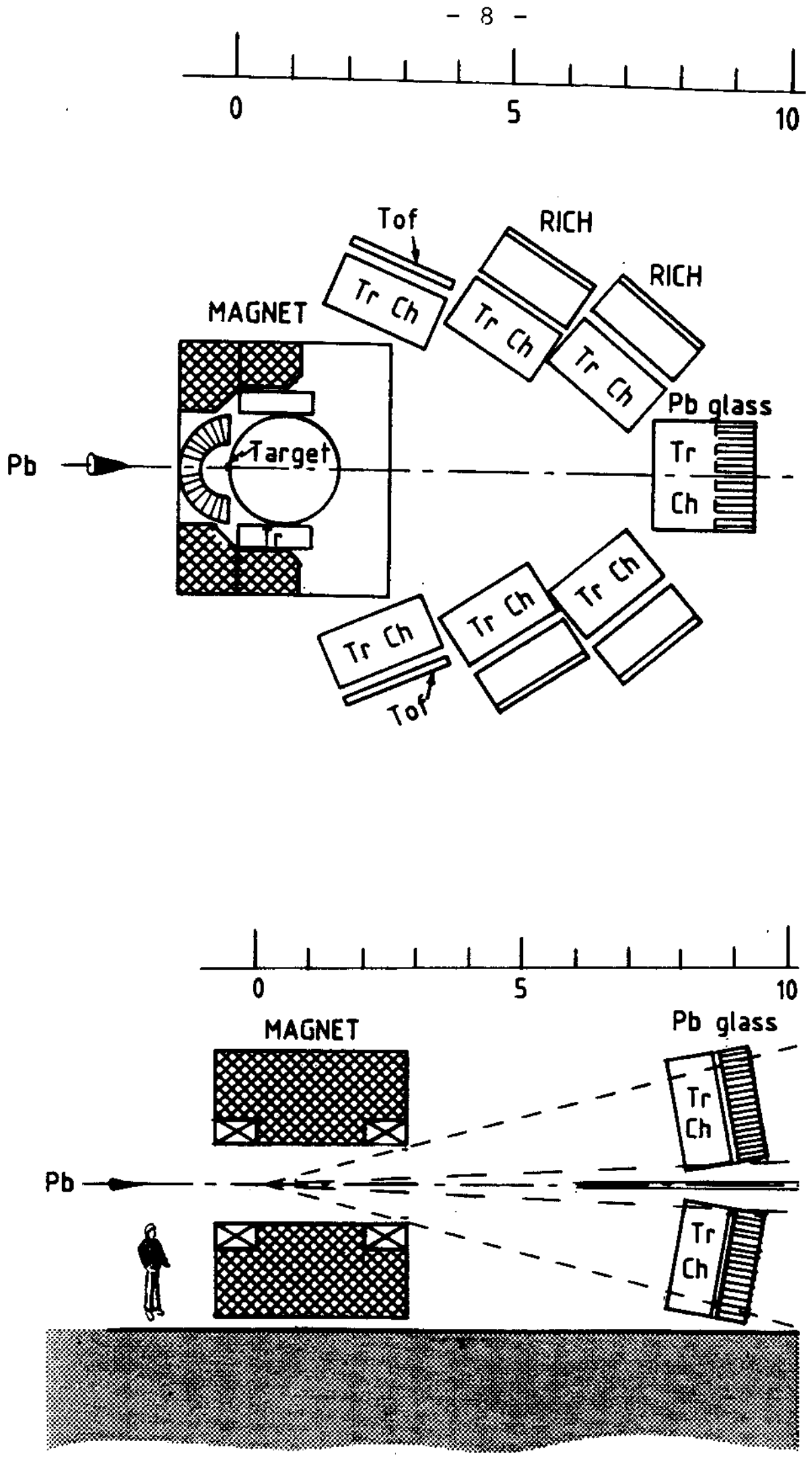

VERTICAL PROJECTION

Fig. 3. Horizontal and vertical projections of a large acceptance detector in which the tracking is done outside of the analyzing vertex magnet where the track density is reduced down to 100 charged particles $/ \mathrm{m}^{2}$. 


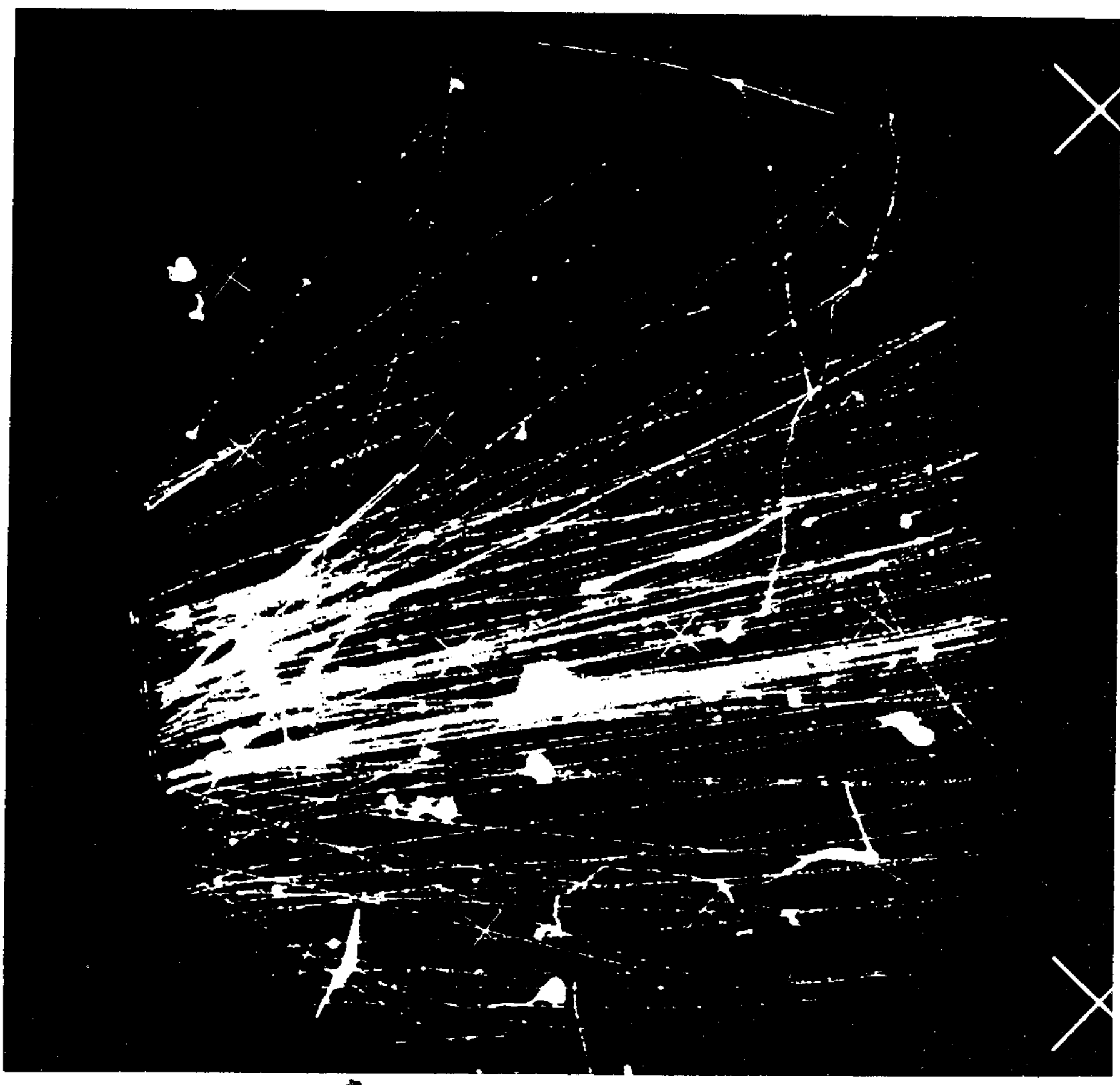

$\rightarrow$

Fig. 4. High multiplicity $v-F e$ interaction as measured by the WA44 Avalanche Chamber. Between the interaction point and the chamber there is a $1 \mathrm{Tm}$ magnet. The tracks are not straight lines due to distortions of the image intensifier system used. The length of the tracks is $1 \mathrm{~m}$. 
particle interferometry, one needs about $1 \mathrm{~m}$ of continuous tracking with submillimeter accuracy. Particles produced in secondary vertices or reinteractions are lost since their momenta cannot be reconstructed by this method. The nature of the tracking detectors that would be the most appropriate has to be determined. A large detector development effort has to be made if one is to cover the many square meters needed to subtend mid - rapidity.

The particle identification for the region of mid-rapidity pions can best be made with RICH counters if one can handle the particle density. For example with a proposed $\mathrm{NaF}$ proximity focused $\mathrm{RICH}$ counter [21], one could identify $\pi^{\prime} \mathrm{s}$ from $.2, \mathrm{~K}^{\prime} \mathrm{s}$ from .5 and $\mathrm{p}^{\prime} \mathrm{s}$ from $1 \mathrm{GeV} / \mathrm{c}$ up to 3 to 5 $\mathrm{GeV} / \mathrm{c}$. Higher momenta can be identified with liquid or gaseous radiators.

For a proximity focused RICH counter, the photons of the Cerenkov radiation are emitted at an angle to the particle's trajectory, given by the velocity of the particle and the index of refraction of the radiator $[\theta=\arccos (1 / \beta n)]$. For normal incidence to the radiator this creates a circular pattern on the detector. Due to the geometry, refraction and total internal reflection, the shapes change to ellipse like' and then to open curves as the incident angle gets smaller (Fig. 5). A simulation of the kind of shapes one would expect to be produced in a $1.5 \times 1.5 \mathrm{~m}^{2} \mathrm{NaF}$ RICH counter at $3.5 \mathrm{~m}$ from the target is shown in Fig. 6. On the left top corner one sees the shapes produced by the particle mix of $\pi^{\prime} \mathrm{s}$ and protons predicted by the Fritiof Lund Monte Carlo for a central ${ }^{32} \mathrm{~S}$ on $\mathrm{Au}$ event. At the top right and bottom left one sees the shapes that would be produced if all the particles were protons or $\pi$ 's respectively. One secs that at the same momentum the pattern produced by protons are smailer and also more often closed than those of the pions since they have a lower velocity. The actual observed pattern of uv photons depends on the thickness of the radiator, on the transmittance of the windows, on the quantum cfficiency of the photosensitive gas of the detector and on the probability to amplify one photoelectron to a measurable signal. Given the very large multiplicities one has to deal with it is essential that the detector measures the space points of the converted photons with good accuracy. Another requirement is to have a two-photon resolution of the order of a few millimetres. One should investigate how the traditional flat drift volumes with MWPC readout [22] would perform. A different type of detector has been reported by the Weizmann Institute - University of Heidelberg group of the NA34 Collaboration [23] consisting of a low pressure multistep avalanche counter read out cither visually or electronically by a large number of pads. A typical ring from a $3 \mathrm{GeV} / \mathrm{c} \mathrm{e} \mathrm{e}^{-}$in a gas radiator, read out by an image intensified CCD camera is shown in Fig. 7. In order to cover many square meters of RICH counters an atmospheric pressure version of such a detector is needed. One finds that such a detector has $100 \%$ efficiency for photoelectrons created in the sensitive volume as well as essentially no noise. Having such a detector in mind with quartz windows and TMEA as a photosensitive agent with a $\mathrm{QE}$ of $38 \%$ and a $0.5 \mathrm{~cm}$ thick $\mathrm{NaF}$ radiator, one can simulate the detector performance for the case discussed previously having 29 charged particles hitting the radiator. The resulting RICH patterns are given by the thick dots in the lower right part of Fig. 6. Clearly one still can identify many of the particles. Up to what multiplicity one can do particle identification with good confidence will depend to a large extent on the background created in such a detector by all the charged particles transversing it.

The analyzing power of the vertex magnet probably will not be sufficient for the density of high momentum $(p>20 \mathrm{GeV} / \mathrm{c}$ ) forward emitted particles to be reduced down to managcable values. Therefore if one is interested in them a second analyzing magnet is needed as shown in Fig. 8.

The dipole magnetic field brakes the cylindrical symmetry of the charged particles by sweeping them away from the central vertical plane. This destroys the calorimetric measurement of $\mathrm{dE}_{\mathrm{T}} / \mathrm{d} \eta$ as is shown in Fig. 9 where the $\mathrm{E}_{\mathrm{T}}$ distribution measured by the NA35 mid-rapidity calorimeters is plotted in the $\eta-\phi$ plane for the case where the Vertex. Magnet was on. On the other hand this sweeping can be of advantage for the detection of photons with fine grained electromagnetic calorimeters since the charged particle flux is greatly reduced in particular in the upper and lower sectors. In order to do $\gamma, \pi^{0}$ and $\eta$ spectroscopy in mid - rapidity with a $\mathrm{Pb}$ - glass calorimeter of the Saphir [24] or the Gams type [25] (Fig. 10), one needs to cover of the order of $16 \mathrm{~m}^{2}$ at a minimum distance of $9 \mathrm{~m}$ from the target. In such a scheme the $\pi^{0}$ reconstruction efficiency and the mass resolution depend more on the two-shower and the position resolution than on the energy resolution for the individual showers. A 

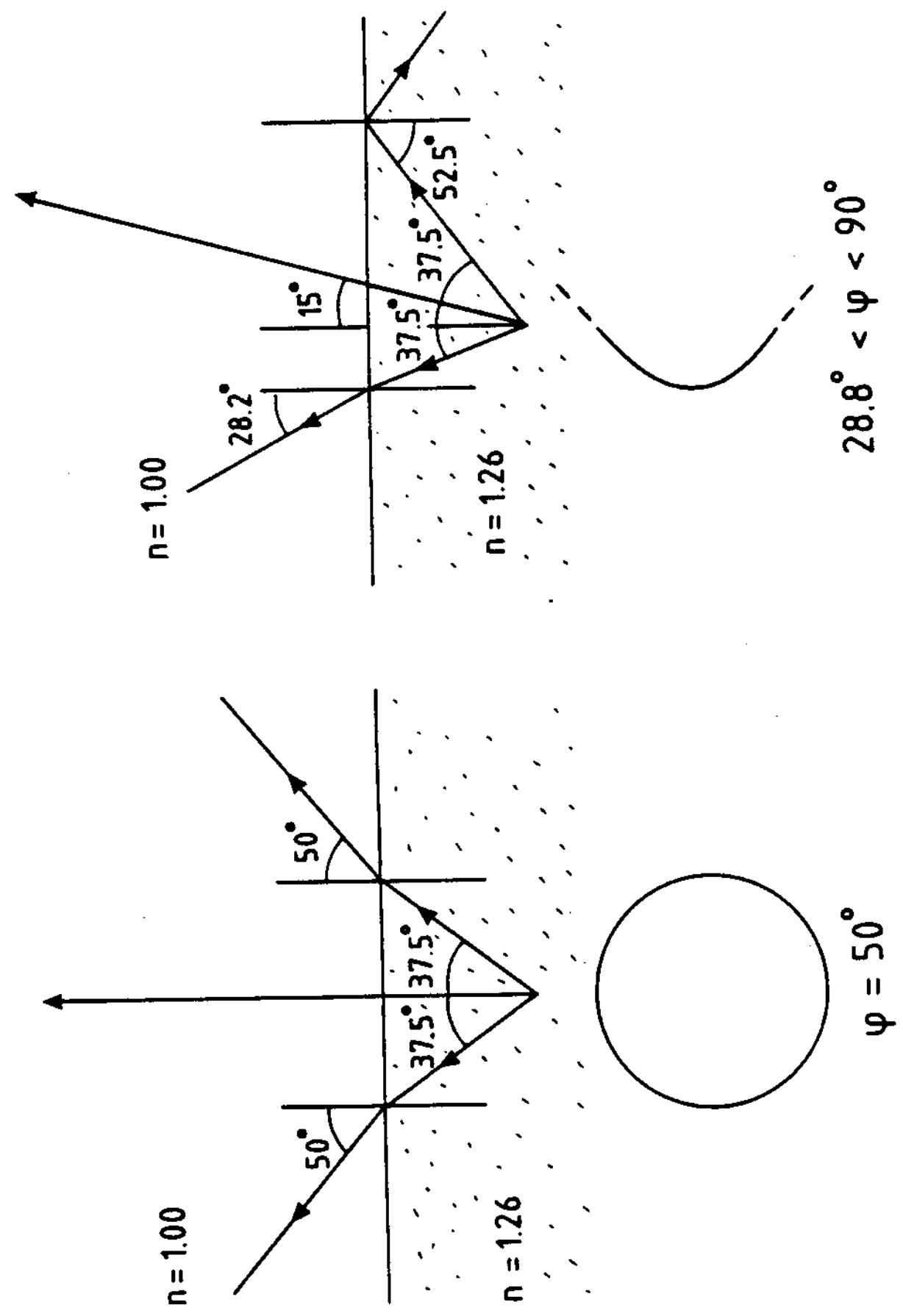

Fig. 5. Image types produced by a proximity focused RICH counter. For nomal incidence of the particle the image is a circle (left graph). For inclined charged particle trajectories one can obtain images that are open paraboloids due to total internal reflection (right graph). 


\section{RICH PATTERNS}
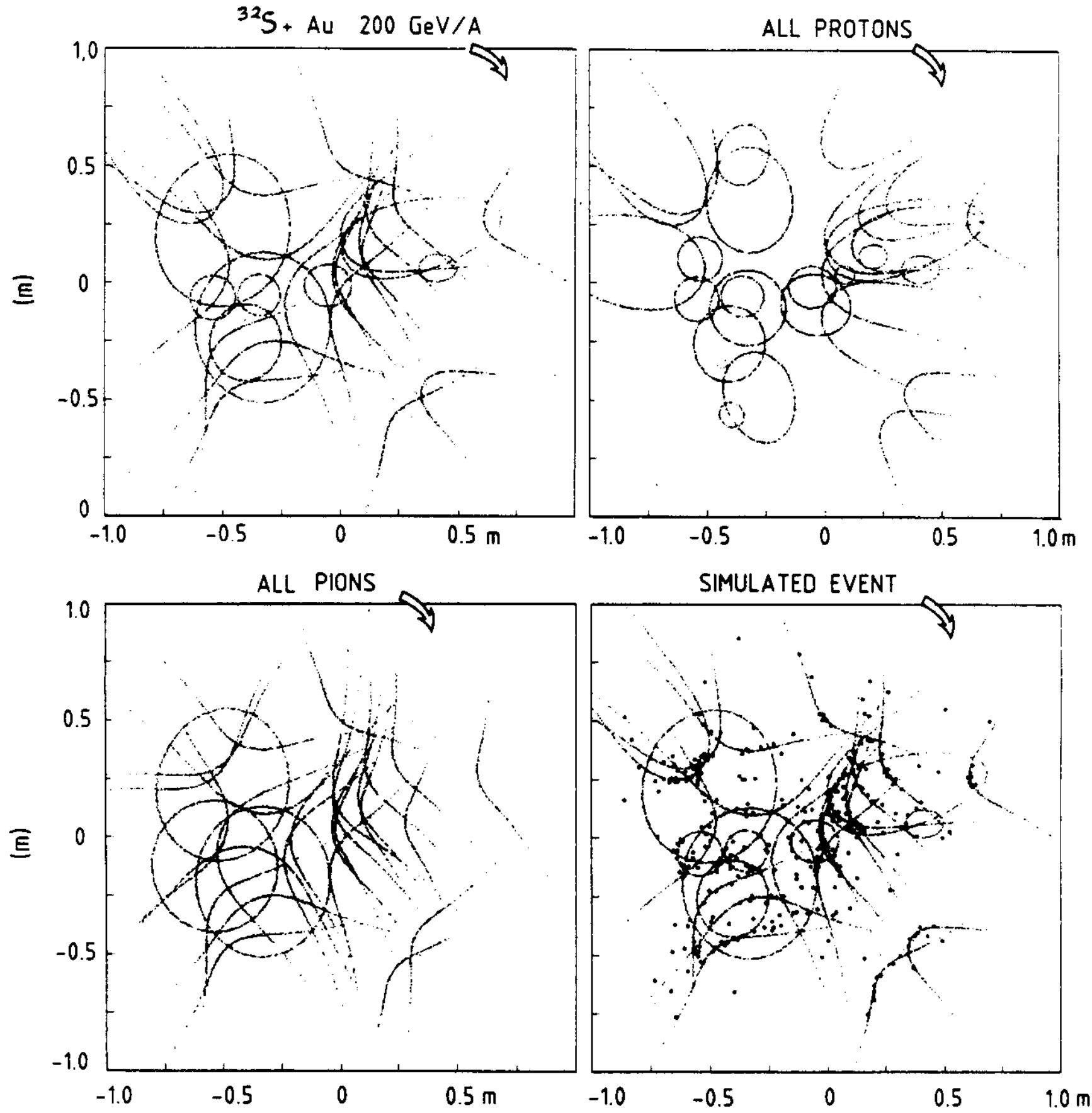

Fig. 6. RICH images produced by a $1.5 \times 1.5 \mathrm{~m}^{2} \mathrm{NaF}$ radiator at $3.5 \mathrm{~m}$ from a $200 \mathrm{GeV} /$ nucleon $32 \mathrm{~S}+\mathrm{Au}$ central collision. At the upper left side for the particle mix given by the event generator. At the right of it the patterns are shown for the case where all the particles are assumed to be protons. And correspondingly at the bottom left if with the same momenta all particles were $\pi$ 's. The dots superimposed on the image at the bottom right represent a simulation of a detector response with $38 \%$ quantum efficiency for uv photons. 

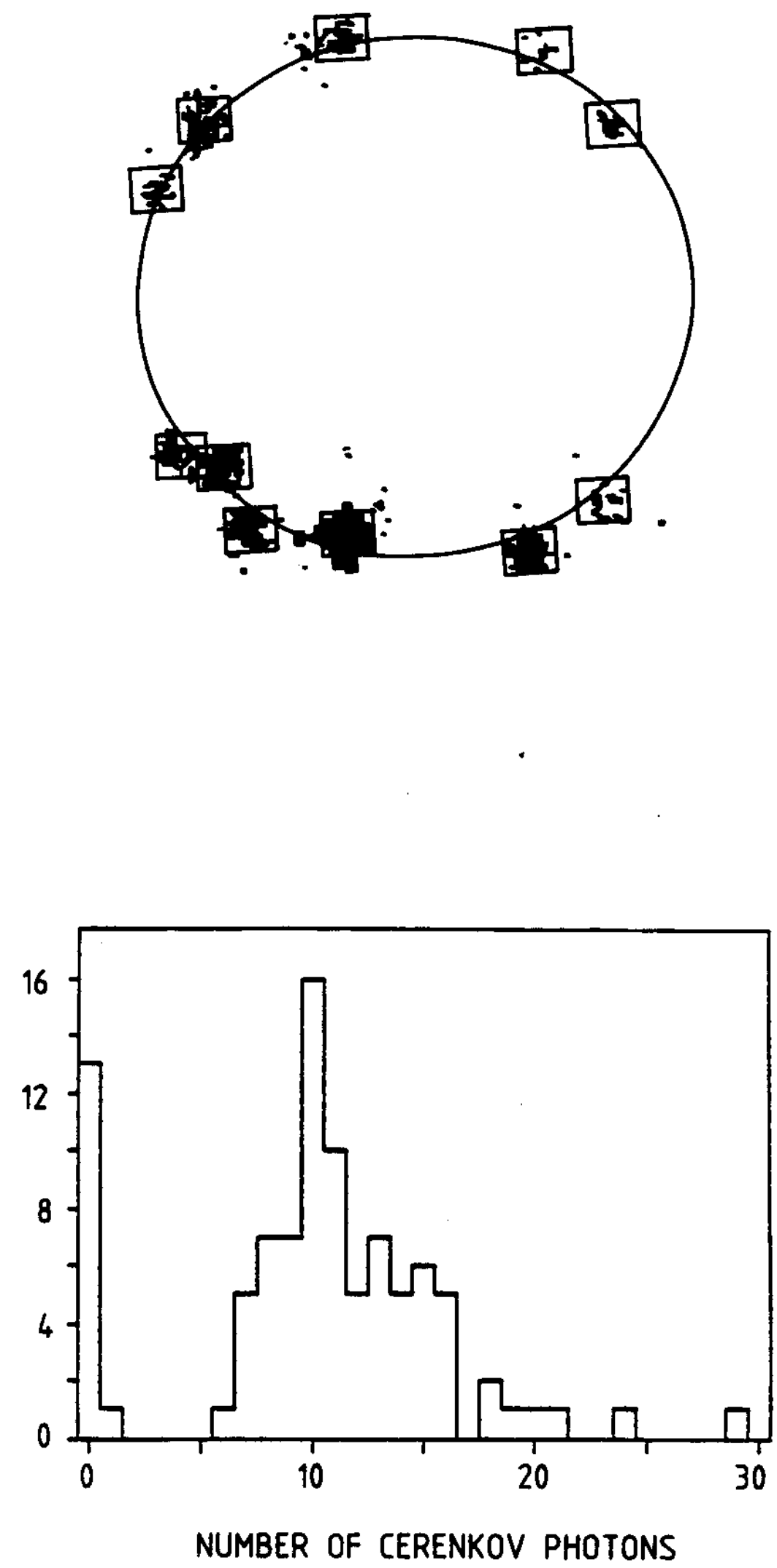

Fig. 7. Measured patterns of a RICH with a low pressure multistep avalanche chamber detecting uv photons. The avalanches in the chamber are recorded optically. One sees the excellent signal/noise ratio. The lower part shows the distribution of the number of recognized light spots per ring. 

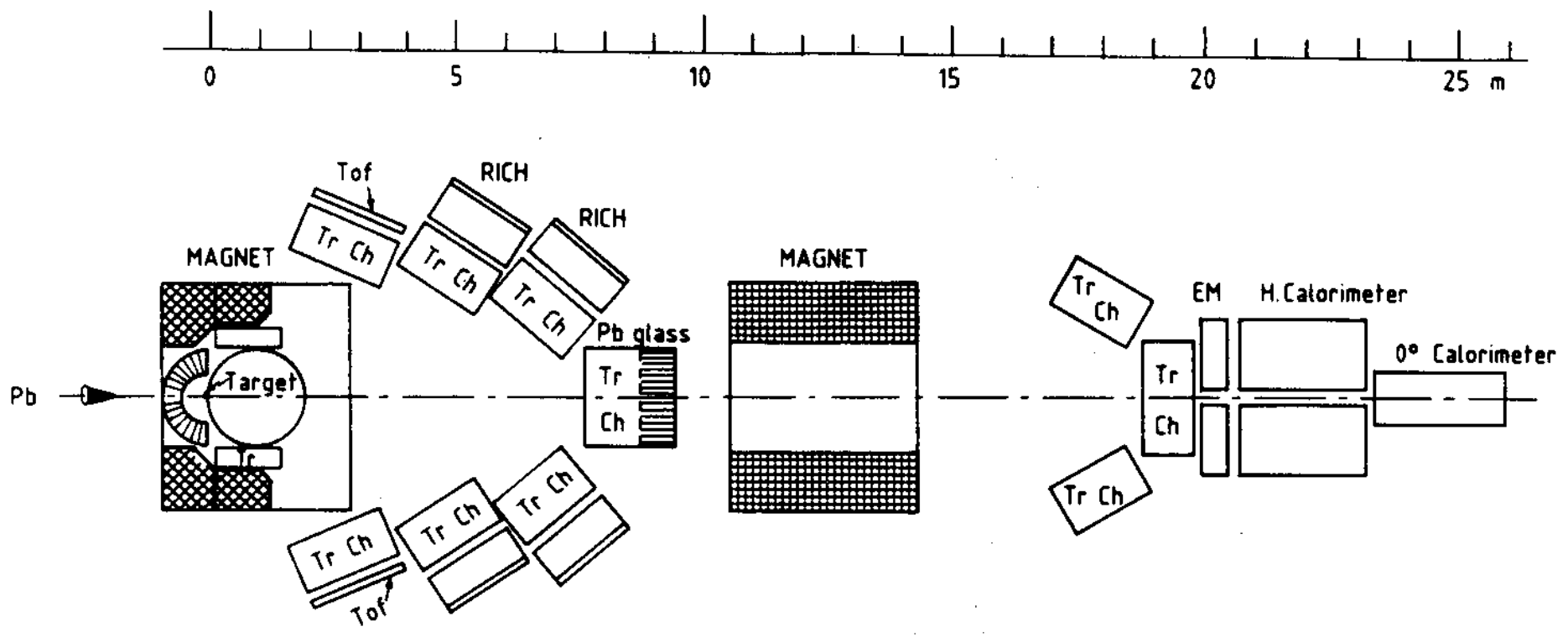

HORIZONTAL PROJECTION

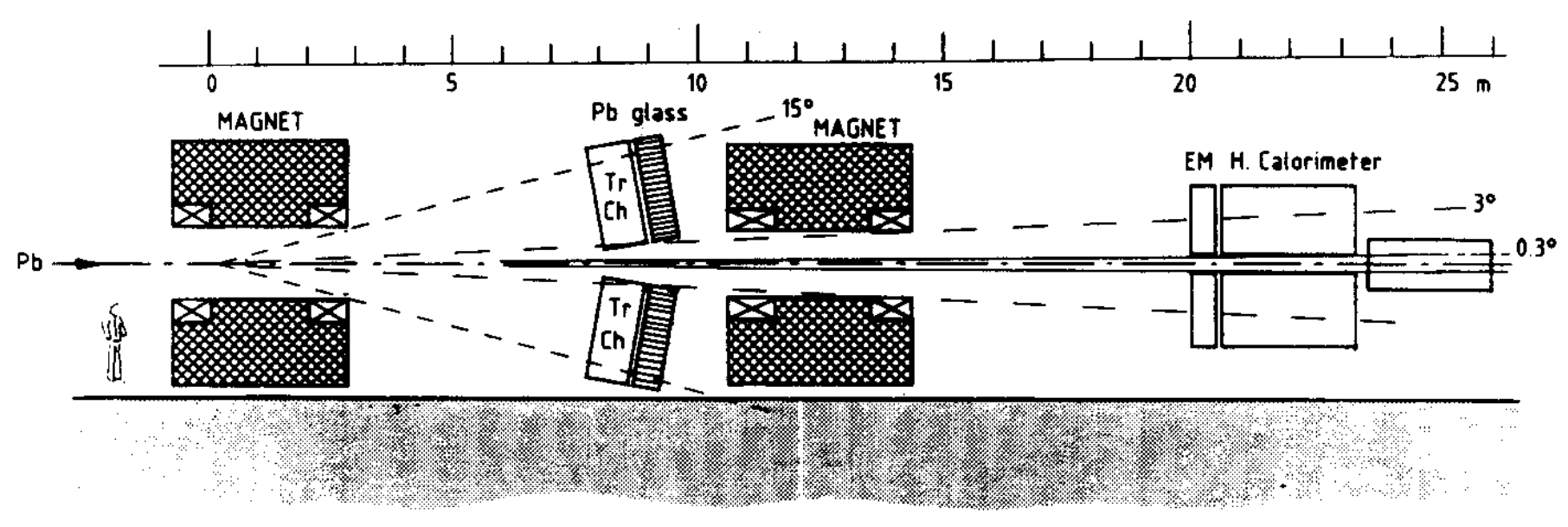

VERTICAL PROJECTION

Fig. 8. In order to detect protons at mid - rapidity and beyond with a large acceptance detector it is necessary to use a second analyzing magnet and further tracking chambers downstream of it. Calorimeters can be used to look at the projectile fragmentation region. 


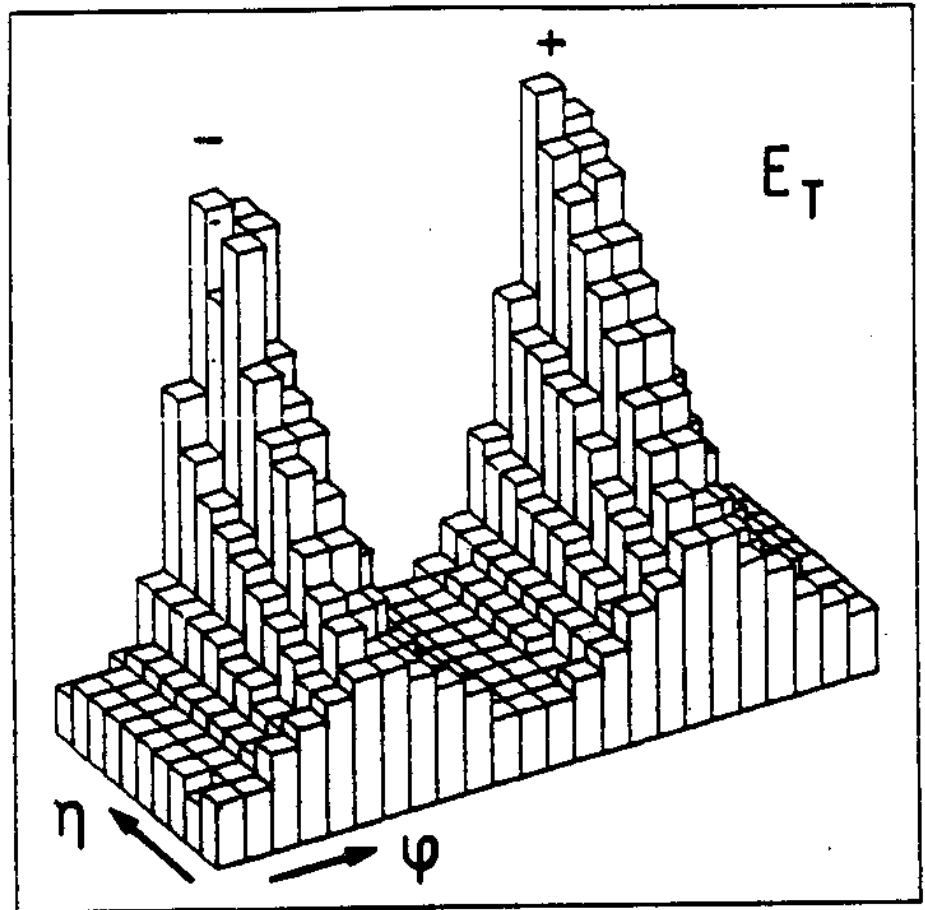

Fig. 9. Shown is the effect of the field of the Vertex Magnet on the NA35 mid-rapidity calorimeters measurement of the transverse energy $\mathrm{E}_{\mathrm{T}}$ for central ${ }^{16} \mathrm{O}+\mathrm{Au}$ collisions.

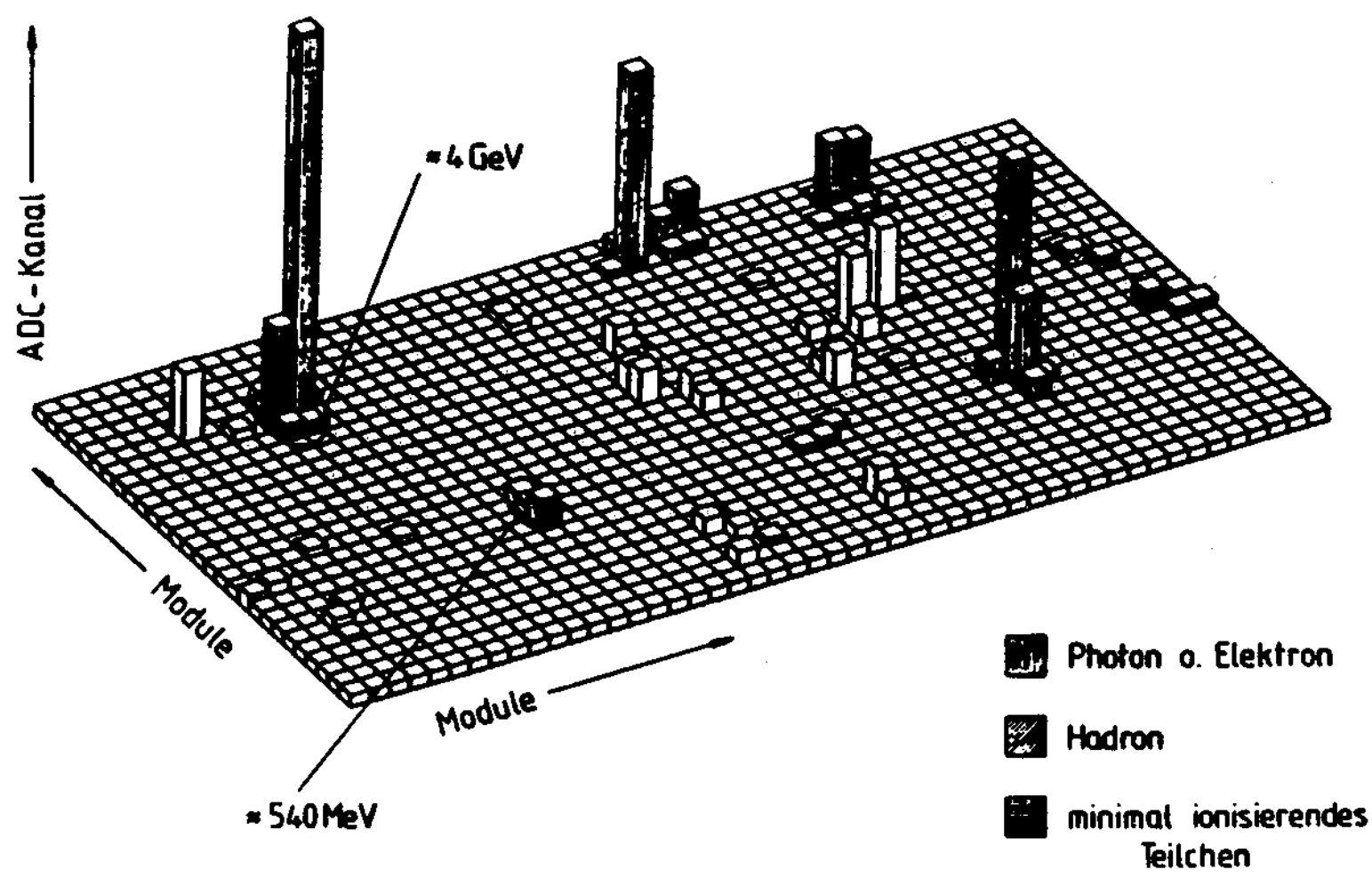

Fig. 10. Event seen by the Saphir Lead-glass array of WA80. A much larger array placed 3 times further away could be used to study central $\mathrm{Pb}+\mathrm{Pb}$ collisions. The sweeping out of charged particles by a vertex magnet and a fine grained preshower detector would improve the $\gamma$ and $\pi^{\circ}$ detection efficiency. 
way to improve over the present design would be to have a preshower detector with better position resolution and good $e / \pi$ rejection and by approaching more closely a tower geometry. One could improve the geometry still using mostly rectangular parallelepipeds by approximating a spherical front face with a mosaic of rectangular arrays. Then only a few other more complicated crystal shapes are needed for the inter array gaps. Again detector development is needed to arrive to the optimal solution.

If it is worthwhile to also do hadron calorimetry over the mid-rapidity region has to be studied. In principle one could subtract the charged hadronic energy as determined by the momentum measurements in the tracking chambers from the calorimeter response and be left with the neutral energy flow whose symmetry around the beam axis would be undistorted. One would like to see a finely segmented hadron calorimeter both in depth and in the transverse direction in order to follow the cores of the showers and obtain a differential energy flow measurement.

As one can see from these simple examples, it is a big challenge to design experiments for the Lead - era. All the detection techniques are pushed to the limit both by the extremely high particle densities and by the need to do kinematically complete measurements.

There are many other techniques that have not been mentioned here that will have to be explored. But it is sure that having a clear aim of what is to be measured, a motivated and dynamic community will find a good solution to a detection problem that in many aspects is of the same magnitude as that of the design of LHC and SSC experiments.

\section{STATUS OF THE LEAD INJECTOR PROJECT.}

The SPS Committee, the CERN Research Board and the Scientific Policy Commitee have favourably considered the lead - ion program as one of the research ficlds to be supported into the 1990's. Such a facility will be unique in the world and would maintain CERN's record of having the beam with the highest energy for many years with $37 \mathrm{TeV}$ extracted $\mathrm{Pb}$ bearns.

A group of accelerator specialists has been set up under the direction of $H$. Haseroth to design the injector structure. It is expected that the injector will be funded as a special project by the national agencies of the groups involved in this field. The group of R. Geller at CEN in Grenoble is working on the design of the ion source, while the GSI Darmstadt has offered its expertise in the design and construction of the accelerating structure. The aim is to get the Lead - Injector Project funded by the end of 1988, to have it constructed over a two year period and to have the first beams by 1991 .

This schedule leaves little time for the conception, design and construction of one or several second generation detectors. Therefore a series of meetings are planned for the first half of 1988 to conceptualize such a detector and to form new collaborations to design and build it. By the end of 1988 it is expected that there will be a large group of people dedicated to this project designing, building and testing prototype detectors, electronics and software. The existing resources in terms of calorimeters, magnets, electronics and computers will have to be pooled together. Nevertheless of the order of 50 MSF will be needed for the construction of such a detector over a period of 3 years. 


\section{CONCLUSIONS}

The future of ultrarelativistic ion physics at CERN looks very bright, both in the near future with the Lead-Injector Project providing beams through the periodic table up to energies of 180 to 225 $\mathrm{GeV} /$ nucleon as well as into the next century when the Large Hadron Collider could have interacting nuclear beams at an energy of $4 \mathrm{TeV} /$ nucleon each.

It is a real challenge to design an experiment that can extract the physics of the high energy density stage produced in central collisions of heavy nuclei with heavy targets (up to $\mathrm{Pb}+\mathrm{Pb}$ ) from the several thousands of produced charged particles and gammas.

\section{References}

[1] L. McLerran, Rev. Mod. Phys. 58(1986)1021 and references therein; Proc. Fifth Int. Conf. on Ultrarelativistic Nucleus-Nucleus Collisions, Asilomar, Calif., eds. L. S. Schroeder and M. Gyulassy, Nucl. Phys. A461(1986).

[2] A. Bamberger et al., NA35 Collaboration, Phys. Lett. B184 (1987)271.

W. Heck et al., NA35 Collaboration, GSI $-88-08$ and this proceedings. Quark Matter 87, Z. Phys.C to be published.

[3] R. Albrecht et al., WA80 Collaboration, Phys. Lett. B199(1987)297.

[4] T. Akesson et al., NA34 Collaboration, CERN-EP/87-176, submitted to Z. Phys. C.

[5] H. Strobele et al., NA35 Collaboration, GSI-88-07 preprint and Quark Matter 87, Z. Phys.C to be published.

[6] I. Lund et al., WA80 Collaboration, Quark Matter 87, Z. Phys.C to be published. R. Albrecht at al., WA80 Collaboration, subrnitted to Phys. Lett. B.

[7] B. Jacak et al., NA34 Collaboration, Quark Matter 87, Z. Phys.C to be published.

[8] H. Lohner et al., WA80 Collaboration, Quark Matter 87, Z. Phys.C to be published.

R. Albrecht et al., WA80 Collaboration, submitted to Phys. Lett. B.

[9] A. Bussiere al., NA38 Collaboration, Quark Matter 87, Z. Phys.C to be published.

[10] T. Humanic et al., NA35 Collaboration Phys. Lett. B to be published and Quark Matter 87, Z. Phys.C to be published.

[11] R. Stock and R. Bock, Univ. Frankfurt preprint 1986.

[12] G.W. London, N.A. McCubbin and R. Stock CERN/SPSC 87-52.

[13] H. Haseroth, A. Lombardi and M. Weiss, CERN preprint PS28 (1987).

[14] G. Vesztergombi et al., NA35 Collaboration, Quark Matter 87, Z. Phys.C to be published.

[15] Y. Miake et al., E802 Collaboration, Quark Matter 87, Z. Phys.C to be published.

[16] H.W. Bartels et al., NA34 Collaboration, Quark Matter 87, Z. Phys.C to be published.

[17] R. Smidt et al., WA80 Collaboration, Quark Matter 87, Z. Phys.C to be published.

[18] I. Otterlund, Quark Matter 87, Z. Phys.C to be published.

[19] L. Ramello et al., NA34 Collaboration, Quark Matter 87, Z. Phys.C to be published.

[20] A. Bamberger et al., NA35 Collaboration, GSI-88-09 preprint submitted to Phys. Lett. B.

[21] R. Amold et al., CERN-EP/87-186. Pres. at the London Conf. on Position Sensitive Detectors (London, 7-11 Sept. 1987).

[22] R. Amold et al., NIM A252(1986) 188.

[23] A. Breskin et al., WIS $-87 / 68 /$ Sept - PH. Pres. at the London Conf. on Position Sensitive Detectors (London, 7-11 Sept. 1987).

[24] H.H. Gutbrod et al., GSI-85-32 preprint and CERN SPSC/85-39/M406.

[25] F. Binon et al., NA12 Collaboration, NIM A248(1986)86. 